\title{
Altered topography of systemic veins following lung tissue resection: the effect on de novo cardiac implantable electronic device implantation
}

\author{
R. Steckiewicz, E.B. Świętoń, K. Rowińska-Berman, P. Stolarz \\ Department of Cardiology, Medical University of Warsaw, Poland
}

[Received: 29 October 2016; Accepted: 17 November 2016]

\begin{abstract}
Venous anomalies discovered on cardiac implantable electronic device (CIED) implantation may hinder both the insertion of cardiac leads and the selection of their optimal intraventricular placement. Such venous anomalies may be a result of congenital vascular defects, e.g. anomalies of the foetal venous system, or be a consequence of earlier cardio- or thoracosurgical procedures. In the case of the latter, the extent of morphometric changes to mediastinal structures may depend on the extent of prior lung tissue resection. This paper presents 3 cases of CIED implantation procedures performed in patients with systemic veins topographically and morphometrically altered post lung surgery. (Folia Morphol 2017; 76, 2: 322-325)
\end{abstract}

Key words: cardiac pacing, pneumonectomy, lobectomy, computed tomography, venography

\section{INTRODUCTION}

The indications for cardiac implantable electronic devices (CIED) have expanded considerably over the years: from pacemakers (PMs) used solely for bradyarrhythmia treatment, through implantable cardioverter defibrillators (ICDs) to control life-threatening arrhythmias, to cardiac resynchronisation therapy (CRT) devices $[3,8]$.

The venous system is used in most of the current CIED implantation procedures for cardiac lead insertion. The conventional techniques of cardiac lead insertion by cephalic vein (CV) or jugular vein (JV) cut-down have been expanded by adding axillary vein (AV) or subclavian vein (SV) puncture with the use of special kits $[2,9]$.

The use of the CV cut-down technique may be limited by congenitally unfavourable venous morphometric parameters or the course of veins altered as a result of surgery. On the other hand, with the
$\mathrm{AV} / \mathrm{SV}$ puncture technique there is a higher risk of accidental damage to nearby anatomical structures resulting in significant complications including pneumothorax, venous or arterial haemorrhaging into the pleural cavity. The risk of these complications increases with atypical spatial topography of the veins used at that stage of a CIED implantation procedure [7].

The effectiveness of subsequent stages of cardiac lead advancement through the venous system is determined by favourable morphometric parameters of further vessels: the brachiocephalic vein (BCV) and the superior vena cava (SVC) as far as the selected lead placement site. Problems at this stage may be due to embryonic systemic venous abnormalities or, alternatively, to past cardio or cardiothoracic surgery $[1,4,6]$.

Due to the fact that such co-occurrence of clinical conditions is very rare, we presented specific alterations of morphometric and topographic parameters

Address for correspondence: R. Steckiewicz, MD, PhD, Department of Cardiology, Medical University of Warsaw, ul. Banacha 1A, 02-097 Warszawa, Poland, tel: +48 22599 2958, e-mail: r.steckiewicz@pro.onet.pl 
of venous vessels observed during de-novo CIED implantation procedures in three patients post lung tissue resection.

\section{CASE REPORTS}

In the period between January 2012 and September 2016, out of 1,901 de-novo CIED implantation procedures performed at our centre, there were three where patients were post thoracic surgery due to malignant neoplasm.

The CIED implantation procedures were conducted according to a standard protocol, with local lignocaine anaesthesia in the infraclavicular region. Device pockets were formed subcutaneously anterior to the pectoral muscle, while cardiac leads were introduced into heart chambers via a transvenous route. Two of these patients received ventricular pacing devices (VVIR mode) and one received an atrioventricular pacing device (DDDR mode). Before their CIED implantation procedures, the patients stayed in an Intensive Care Unit, where their heart rates were effectively controlled with medication, precluding any need for temporary cardiac pacing.

\section{CIED IMPLANTATION POST RIGHT TOTAL PNEUMONECTOMY}

Case 1. A 72-year-old male, qualified for a PM implantation due to Adams-Stokes episodes resulting from atrioventricular conduction disorder with bradyarrhythmias and persistent atrial fibrillation. The patient had a history of right total pneumonectomy (in 1991) due to lung cancer. On April 6, 2012, there was an unsuccessful attempt at PM implantation on the right side. Four days later, a device with VVI pacing mode (a Talos SR pacemaker [Biotronik] with a 507658 cardiac lead [Medtronic]) was implanted on the left side. Unfavourable CV morphometry prompted cardiac lead insertion via SV puncture (Fig. 1A).

Case 2. An 80-year-old male, qualified to receive a PM due to Adams-Stokes episodes caused by episodes of bradyarrhythmia resulting from sinus node dysfunction. The patient had had a pneumonectomy many years earlier due to squamous cell carcinoma of the right lung. On July 27, 2012, the patient received a Talos SR pacemaker (VVI mode) (Biotronik) with a Setrox S60 cardiac lead introduced via left SV puncture (Fig. 2B).

The course of the CV prevented lead advancement via a cut-down technique. Despite contrast-enhanced vessel imaging, attempts at SV puncture resulted in several instances of SV puncture, with no clinical sequelae.

\section{CIED IMPLANTATION POST LEFT INFERIOR LOBECTOMY}

Case 3. A 69-year-old male, qualified to undergo PM implantation due to an abortive form of AdamsStokes attacks during recorded electrocardiographic episodes of sinus bradycardia with trifascicular block: prolonged PQ (0.22 s), left anterior hemiblock, and right bundle branch block. The patient reported a previous surgical treatment of thymoma with unilateral, lower left lobectomy in 1995. On September 5, 2016, there was a failed attempt to implant a CIED on the left side (Fig. 2A, B). A computed tomography (CT) examination conducted on the next day revealed the cause of the failure: the lumen of the left BCV misshaped by the aortic arch, in the form of segmental compression of the vein with local lumen reduction to $4.0 \mathrm{~mm}$; at the same time, the images confirmed favourable lead insertion conditions on the right side (Fig. 3A, B). On September 7, 2016, an Etrinsa 6 DR-T pacemaker (Biotronik) was implanted with a Solia $S$ 60 ventricular lead inserted via CV cut-down and a Solia S 53 atrial lead inserted via SV puncture.

\section{CONCLUSIONS}

The cases presented here are characterised by a combination of medical, pulmonological, and cardiological procedures that had occurred in a random order. The diagnosed neoplastic conditions required various amounts of lung tissue to be resected. The degree of topographic alterations of mediastinal structures and veins, observed also during CIED implantation procedures, correlates with the volume of resected lung tissue: pneumonectomy vs. lobectomy [1].

Venography with the use of contrast to visualise $\mathrm{CV}, \mathrm{AV}$, and SV lumina can only to some extent help with the initial decision on the side of CIED implantation and the technique of venous access. In the cases presented here it is difficult to unequivocally demonstrate a correlation between the effect of past thoracic surgery procedures on anatomical features of the $\mathrm{CV}$ that proved unfavourable for cardiac lead insertion. The extent of data on mediastinal vessels obtained via venography proved to be insufficient. In the case of congenital defects and post-thoracicsurgery patients who require catheter and/or cardiac lead insertion into the venous system, venography can be supplemented with a diagnostically valid CT examination [11].

In the cases presented here, after total pneumonectomy the course of cardiac leads on fluoroscopy, 


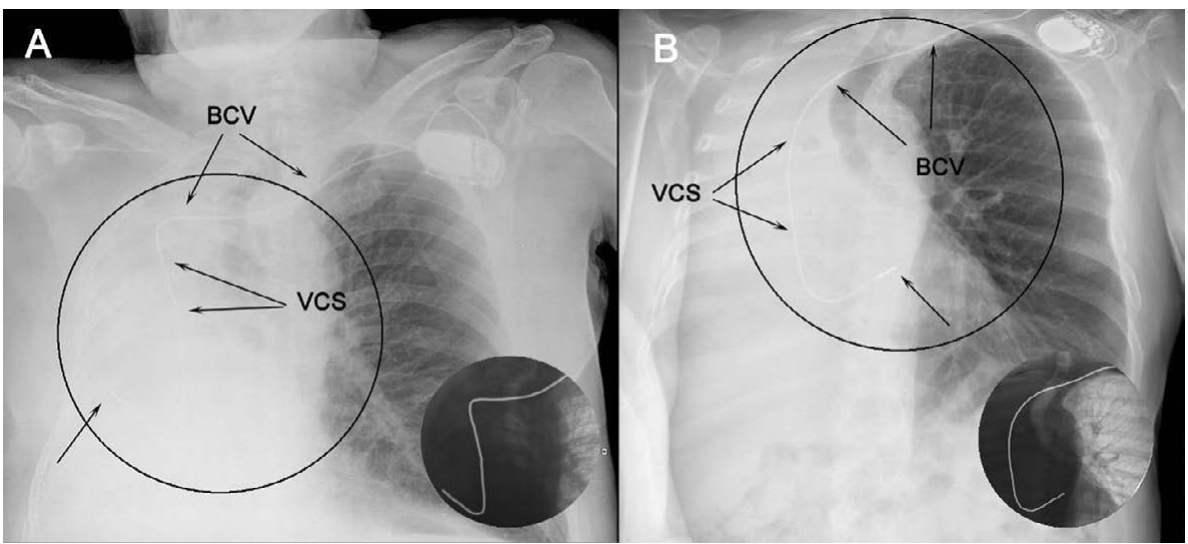

Figure 1. A, B. Postero-arterial view chest $X$-ray images of both patients: the heart and the mediastinum with the right superior vena cava are shifted to the right. In both cases, the cardiac lead is moulded by the veins used for its insertion, which shows a significantly altered topography of those vessels. The lead tip placement in the right ventricle also clearly differs from its conventional location (single black arrow); VCS — vena cava superior; BCV — brachiocephalic vein.

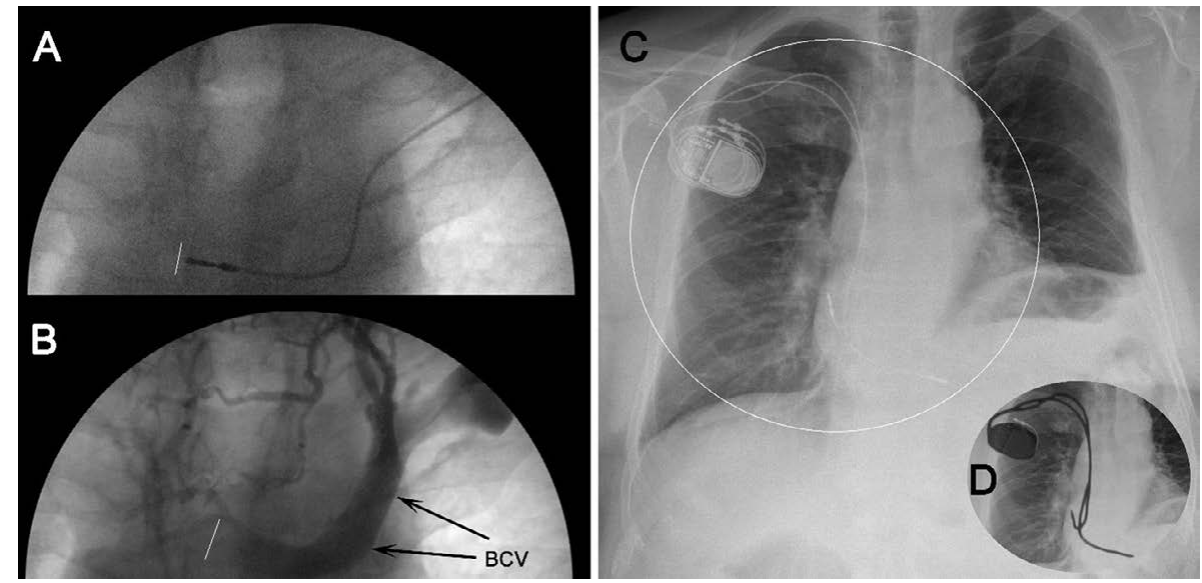

Figure 2. A. A fluoroscopic image of the cardiac lead blocked during its advancement along the brachiocephalic vein (BCV); B. Contrast administration helped visualise contrast flow impairment in the medial segment of the BCV with contrast-enhanced compensatory collateral circulation; C, D. The visualised course of cardiac leads of a DDD device implanted on September 7, 2016.

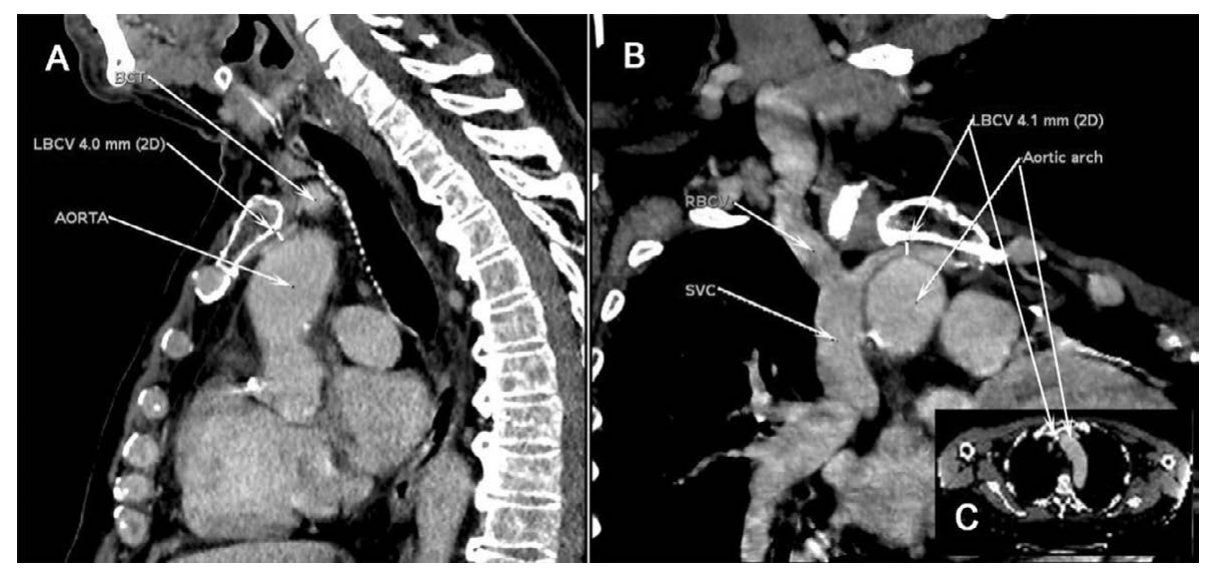

Figure 3. Computed tomography (CT): topographies of the brachiocephalic vein (BCV) and superior vena cava (SVC) in relation to the adjacent anatomical structures of the chest. A. The left BCV (LBCV) segmentally compressed by the aortic arch; B. A frontal reconstruction adjusted to visualise the BCV-right superior vena cava confluence; $C$. The relation of the evaluated structures in an axial CT plane. 
significantly documents a clear "shift" of the left BCV and its drainage point into the SVC to the right with respect to the typical topography of those vessels in the mediastinum. Nonetheless, cardiac leads were successfully advanced through this venous segment. Despite a much lesser extent of tissue destruction, the resection of a single lobe of the left lung still made it impossible to advance a cardiac lead through the left BCV.

In the first two of the three cases presented here fluoroscopy provided only a limited amount of data as to the precise location of leads in heart chambers. Therefore, the operators generally relied on electrophysiological parameters: the endocardial cardiac stimulation threshold and the recorded intracardiac signal magnitude.

The sporadic co-occurrence of events from past medical history and the course of CIED implantation procedures did not allow the authors to draw farreaching conclusions; however, some of the comments regarding the performed procedures may be useful in similar cases.

Expanded indications for CIED implantation in combination with the observed increased incidence of neoplastic diseases that require surgical treatment involving lung tissue resection, are sure to increase co-occurrence of similar cases $[5,10]$.

In the case of procedures performed in post-thoracic-surgery patients and requiring catheter and/or lead insertion into the venous system, irrespective of the standard venography, there seems to be an indication for a prior $\mathrm{CT}$ vessel reconstruction adjusted to the type of the planned invasive procedure.

\section{REFERENCES}

1. Bazwinsky-Wutschke $I$, Paulsen $F$, Stövesandt $D$, et al. Anatomical changes after pneumonectomy. Ann Anat - Anat Anz. 2011; 193(2): 168-172, doi: 10.1016/j. aanat.2011.01.002.

2. Bongiorni MG, Proclemer A, Dobreanu D, et al. Scientific Initiative Committee, European Heart Rhythm Association.
Preferred tools and techniques for implantation of cardiac electronic devices in Europe: results of the European Heart Rhythm Association survey. Europace. 2013; 15(11): 1664-1668, doi: 10.1093/europace/eut345, indexed in Pubmed: 24170423.

3. Brignole M, Auricchio A, Baron-Esquivias G, et al. 2013 ESC Guidelines on cardiac pacing and cardiac resynchronization therapy: The Task Force on cardiac pacing and resynchronization therapy of the European Society of Cardiology (ESC). Developed in collaboration with the European Heart Rhythm Association (EHRA). Europace. 2013; 15(8): 1070-1118, doi: 10.1093/europace/eut206.

4. Demos TC, Posniak HV, Pierce KL, et al. Venous anomalies of the thorax. AJR Am J Roentgenol. 2004; 182(5): 1139-1150, doi: 10.2214/ajr.182.5.1821139, indexed in Pubmed: 15100109.

5. Didkowska J. Epidemiologia nowotworów złośliwych w Polsce. Podstawy onkologii klinicznej. CMKP, Warszawa 2011.

6. Kahkouee S, Sadr M, Pedarzadeh E, et al. Anomalous left brachiocephalic vein: important vascular anomaly concomitant with congenital anomalies and heart diseases. Folia Morphol. 2016 [Epub ahead of print], doi: 10.5603/FM.a2016.0031, indexed in Pubmed: 27830886.

7. Kirkfeldt RE, Johansen JB, Nohr EA, et al. Pneumothorax in cardiac pacing: a population-based cohort study of 28,860 Danish patients. Europace. 2012; 14(8): 1132-1138, doi: 10.1093/europace/eus054, indexed in Pubmed: 22431443.

8. Kuck $\mathrm{K}-\mathrm{H}$, Hindricks $\mathrm{G}$, Padelleti $\mathrm{L}$, et al. The EHRA White Book. 2015: 401-410.

9. Lau EW. Upper body venous access for transvenous lead placement: review of existent techniques. Pacing Clin Electrophysiol. 2007; 30(7): 901-909, doi: 10.1111/j.15408159.2007.00779.x, indexed in Pubmed: 17584273.

10. Raatikainen MJ, Arnar DO, Zeppenfeld K, et al. Statistics on the use of cardiac electronic devices and electrophysiological procedures in the European Society of Cardiology countries: 2014 report from the European Heart Rhythm Association. Europace. 2015; 17 (Suppl 1): i1-75, doi: 10.1093/europace/euu300, indexed in Pubmed: 25616426.

11. Whitten CR, Khan S, Munneke GJ, et al. A diagnostic approach to mediastinal abnormalities. Radiographics. 2007; 27(3): 657-671, doi: $10.1148 / \mathrm{rg} .273065136$, indexed in Pubmed: 17495284. 